\title{
Practical bioethics
}

\author{
Michael J. Selgelid ${ }^{1}$
}

The emergence of Practical Ethics during the second half of the twentieth century reflected the aim of scholars with expertise in ethics to conduct socially valuable work by (1) focusing on real-world problems of pressing practical concern and (2) providing competent practical guidance about how such matters should be dealt with. Bioethics, or so I always thought anyway, is meant to be the sub-discipline of Practical Ethics that does this kind of work in the context of the life sciences, biomedical sciences, and healthcare, etc.

The actual practicality of some (or perhaps much) bioethics discourse, on the other hand, might be considered dubious and/or quite limited. Rather than being pressing practical problems of our times - or even practical problems we are likely to be faced with any time soon (if ever)—numerous subjects of bioethics discourse involve what are currently science fiction scenarios. Some of these are, arguably at least, quite far fetched.

I would by no means want to deny that it is practically valuable to conduct ethical analysis of new technologies that will likely or possibly arise in the future. This is at least partly because the future is hard to predict, and some technologies become realities earlier than expected. Before Dolly the sheep was cloned in the 1990s, for example, experts commonly doubted that mammalian cloning would become technically possible in the near future. Thinking about the ethics of possible future technologies may also be useful to guide the direction of research and development. Ethical analysis, that is, may provide reasons to steer research towards some, and away from other, areas of technological development. Waiting for possible new technologies to become realities, furthermore, may be imprudent-because ethics would then (as is commonly said about the law) forever be lagging behind and

Michael J. Selgelid

michael.selgelid@monash.edu

1 Centre for Human Bioethics, Monash University, Clayton, VIC 3800, Australia 
trying to catch up. Ethical analysis might also then come too late to prevent damage that has already been done.

Something would be amiss, on the other hand, if attention to issues that are not such pressing problems come at the cost of neglect to those that are most, or more, important. It is doubtful that the most practically important bioethical issues have actually been getting the most (or even adequate) attention in contemporary bioethics discourse - and/or, more generally speaking, it is doubtful that bioethical attention to issues in bioethics literature/discourse is proportional to their bioethical importance. (This, admittedly, warrants empirical study.)

There are numerous reasons why, if true, this might be the case. One reason might be that many bioethicists are motivated to think and write about the issues that they consider to be most interesting, or that they perceive to be most philosophically difficult, rather than those that are necessarily the most pressing and practically important. If so, then that might be fair enough. I would not want to claim that all bioethicists should focus on the most (or highly) practical issues any more than I would want to claim that all ethicists should engage in practical rather than more theoretical/abstract ethics.

It is not obvious, on the other hand, that the most practical issues should generally be considered less interesting or difficult than less practical issues. To the contrary, the richness of real world context can often make current pressing issues all the more interesting and difficult (whereas the idealization and/or simplification involved in abstraction away from reality may often make problems more tractable/ easy rather than difficult).

The sheer difficulty of some of the most important practical problems (e.g., given all the empirical facts and other complexities involved) might be a particularly good explanation of why they don't receive more attention, especially when they arrive rather unexpectedly (and relevant scholars do not already have the background expertise required to address them adequately, as they have often spent careers focused on other kinds of things).

Regardless of explanation, it would be tragic if the discipline of bioethics fails to adequately address what are actually and currently the most practically important issues of bioethical concern. If it fails to provide the best possible analysis of the most practically important issues of bioethical concern, the discipline of bioethics would have failed to do its job. Everyone working in bioethics need not focus on actually (or highly) practical issues, but some — and enough-surely should.

Perhaps the time has thus come to explicitly establish, promote, and distinguish Practical Bioethics as a sub-discipline of Bioethics more generally-just as Practical Ethics was previously established, promoted, and distinguished as a special branch of Ethics. 